\title{
575.
}

\section{ON A SPECIAL QUARTIC TRANSFORMATION OF AN ELLIPTIC FUNCTION.}

[From the Quarterly Journal of Pure and Applied Mathematics, vol. xII. (1873), pp. 266-269.]

IT is remarked by Jacobi that a transformation of the order $n^{\prime} n^{\prime \prime}$ may lead to a modular equation

$$
\frac{\Delta^{\prime}}{\Delta}=\frac{n^{\prime}}{n^{\prime \prime}} \frac{K^{\prime}}{K}
$$

and in particular when $n^{\prime}=n^{\prime \prime}$, or the order is square, then the equation may be $\frac{\Delta^{\prime}}{\Delta}=\frac{K^{\prime}}{K}$; viz. that instead of a transformation we may have a multiplication. A quartic transformation of the kind in question may be obtained as follows: writing

$$
X=\left(a, b, c, d, e \gamma(x, 1)^{4}=a(x-\alpha)(x-\beta)(x-\gamma)(x-\delta),\right.
$$

$H$ the Hessian, $\Phi$ the cubi-covariant, $I$ and $J$ the two invariants, then there is a well known quartic transformation

leading to

$$
z=\frac{2 H}{X}
$$

$$
\frac{d z}{\sqrt{ }(Z)}=\frac{2 \sqrt{ }(-2) d x}{\sqrt{ }(X)}
$$

where $Z=z^{3}-I z+2 J$. In fact we have

that is,

$$
Z=\frac{2}{X^{3}}\left(4 H^{3}-I H^{2} X+J X^{3}\right), \quad=\frac{-2}{X^{3}} \Phi^{2},
$$

$$
\sqrt{ }(Z)=\frac{\sqrt{ }(-2) \Phi}{X^{2}} \sqrt{ }(X)
$$


so that, by Jacobi's general principle, it at once appears that we have a transformation of the form in question.

Now we may establish a linear transformation

$$
z=\frac{p y+q}{y-\delta}
$$

such that to the roots $z_{1}, z_{2}, z_{3}$ of the equation $z^{3}-I z+2 J=0$ correspond the values $\alpha, \beta, \gamma$ of $y$; and this being so, we have between $y, z$ the relation

$$
\frac{d z}{\sqrt{ }(Z)}=\frac{\sqrt{ }(-2) d y}{\sqrt{ }(Y)}
$$

where $Y=a(y-\alpha)(y-\beta)(y-\gamma)(y-\delta),=(a, b, c, d, e \gamma y, 1)^{4}$; that is, we have

such that

$$
\frac{p y+q}{y-\delta}=\frac{2 H}{X}
$$

$$
\frac{d y}{\sqrt{ }(Y)}=\frac{2 d x}{\sqrt{ }(X)}
$$

which is a quartic transformation giving a duplication of the integral. The foundation of the theorem is that we can determine $p, q$ in such wise that the functions

$$
\frac{p \alpha+q}{\alpha-\delta}, \quad \frac{p \beta+q}{\beta-\delta}, \quad \frac{p \gamma+q}{\gamma-\delta}
$$

shall be the roots $z_{1}, z_{2}, z_{3}$ of the equation $z^{3}-I z+2 J=0$. For writing

and observing the equations

$$
\begin{aligned}
& A=(\beta-\gamma)(\alpha-\delta), \\
& B=(\gamma-\alpha)(\beta-\delta), \\
& C=(\alpha-\beta)(\gamma-\delta),
\end{aligned}
$$

$$
I=\frac{a^{2}}{24}\left(A^{2}+B^{2}+C^{2}\right), \quad=-\frac{a^{2}}{12}(B C+C A+A B),
$$

(since $A+B+C=0$ ) and

$$
2 J=-\frac{a^{3}}{216}(B-C)(C-A)(A-B)
$$

the equation in $z$ is

$$
\left\{z-\frac{1}{6} a(B-C)\right\}\left\{z-\frac{1}{6} a(C-A)\right\}\left\{z-\frac{1}{6} a(A-B)\right\},
$$

and the equations for the determination of $p, q$ thus are

$$
\begin{aligned}
& p \alpha+q=\frac{1}{6} a(\alpha-\delta)(B-C),=\frac{1}{6} a(\alpha-\delta)\{2(\alpha \delta+\beta \gamma)-(\alpha+\delta)(\beta+\gamma)\}, \\
& p \beta+q=\frac{1}{6} a(\beta-\delta)(C-A),=\frac{1}{6} a(\beta-\delta)\{2(\beta \delta+\gamma \alpha)-(\beta+\delta)(\gamma+\alpha)\}, \\
& p \gamma+q=\frac{1}{6} a(\gamma-\delta)(A-B),=\frac{1}{6} a(\gamma-\delta)\{2(\gamma \delta+\alpha \beta)-(\gamma+\delta)(\alpha+\beta)\},
\end{aligned}
$$


giving

$$
\begin{aligned}
& p=\frac{1}{6} a\left\{-3 \delta^{2}+2 \delta(\alpha+\beta+\gamma)-\beta \gamma-\gamma \alpha-\alpha \beta\right\}, \\
& q=\frac{1}{6} a\left\{\delta^{2}(\alpha+\beta+\gamma)-2 \delta(\beta \gamma+\gamma \alpha+\alpha \beta)+3 \alpha \beta \gamma\right\}
\end{aligned}
$$

or, as these may also be written

$$
\begin{aligned}
& p=\frac{1}{6} a\{(\beta-\delta)(\gamma-\delta)+(\gamma-\delta)(\alpha-\delta)+(\alpha-\delta)(\beta-\delta)\}, \\
& q=\frac{1}{6} a\{\alpha(\beta-\delta)(\gamma-\delta)+\beta(\gamma-\delta)(\alpha-\delta)+\gamma(\alpha-\delta)(\beta-\delta)\}
\end{aligned}
$$

and observe also

$$
p \delta+q=\frac{1}{2} a(a-\delta)(\beta-\delta)(\gamma-\delta) .
$$

Taking $X$ in the standard form $=\left(1-x^{2}\right)\left(1-k^{2} x^{2}\right)$, and writing

$$
\gamma=-1, \quad \delta=1, \quad \alpha=+\frac{1}{k}, \quad \beta=-\frac{1}{k},
$$

we have

$$
\begin{gathered}
z=\frac{p y+q}{y-1}=\frac{-\frac{1}{6}\left\{2 k^{2}\left(1+k^{2}\right)\left(1+k^{2} x^{4}\right)+\left(1-10 k^{2}+k^{4}\right) x^{2}\right\}}{\left(-x^{2}\right)\left(1-k^{2} x^{2}\right)}, \\
A=-1+\frac{2}{k}-\frac{1}{k^{2}}, \\
B=1+\frac{2}{k}+\frac{1}{k^{2}}, \\
C=\quad-\frac{4}{k} ; \\
z_{1}=\frac{1}{6}\left(1+6 k+k^{2}\right), \\
z_{2}=\frac{1}{6}\left(1-6 k+k^{2}\right), \\
z_{3}=-\frac{1}{3}\left(1+k^{2}\right) ; \\
Z=z^{3}-\frac{1}{12}\left(1+14 k^{2}+k^{4}\right) z+\frac{1}{108}\left(1+k^{2}\right)\left(1-34 k^{2}+k^{4}\right) \\
=\left(z-z_{1}\right)\left(z-z_{2}\right)\left(z-z_{3}\right), \\
p=\frac{1}{6}\left(1-5 k^{2}\right), \quad q=\frac{1}{6}\left(5-k^{2}\right), \quad p+q=1-k^{2} ;
\end{gathered}
$$

giving as they should do

$$
z_{1}=\frac{\frac{p}{k}+q}{\frac{1}{k}-1}, \quad z_{2}=\frac{-\frac{p}{k}+q}{-\frac{1}{k}-1}, \quad z_{3}=\frac{-p+q}{-2}
$$

Write for shortness

$$
-\frac{1}{6}\left\{2 k^{2}\left(1+k^{2}\right)\left(1+x^{4}\right)+\left(1-10 k^{2}+k^{4}\right) x^{2}\right\}=Q
$$

so that

$$
\frac{p y+q}{y-1}=\frac{Q}{X}
$$

C. IX. 
then

$$
\begin{aligned}
& \frac{Q}{X}-z_{1}=\frac{p+q}{k-1} \cdot \frac{k y-1}{y-1} \\
& \frac{Q}{X}-z_{2}=\frac{p+q}{k+1} \cdot \frac{k y+1}{y+1} \\
& \frac{Q}{X}-z_{3}=\frac{p+q}{2} \cdot \frac{y+1}{y-1}
\end{aligned}
$$

The last of these is

$$
\frac{1-k^{2}}{2} \frac{y+1}{y-1}=\frac{\frac{1}{2}\left(1-k^{2}\right)^{2} x^{2}}{\left(1-x^{2}\right)\left(1-k^{2} x^{2}\right)},
$$

that is,

$$
\frac{y+1}{y-1}=\frac{\left(1-k^{2}\right) x^{2}}{\left(1+x^{2}\right)\left(1-k^{2} x^{2}\right)},
$$

from which the foregoing equation

$$
\frac{d y}{\sqrt{ }(Y)}=\frac{2 d x}{\sqrt{ }(X)}
$$

may be at once verified. 